Volume 9, No.1.1, 2020

International Journal of Advanced Trends in Computer Science and Engineering

Available Online at http://www.warse.org/IJATCSE/static/pdf/file/ijatcse0291.12020.pdf

https://doi.org/10.30534/ijatcse/2020/0291.12020

\title{
Swine Management System in PLF Integrating Image Processing for Weight Monitoring
}

\author{
Anrem J. Balontong', Bobby Gerardo ${ }^{2}$, Ruji P. Medina ${ }^{3}$ \\ ${ }^{1}$ Technological Institute of the Philippines, Quezon City, Philippines, anrem.balontong@gmail.com \\ ${ }^{1}$ Western Institute of Technology, Iloilo City, Philippines, anrem.balontong@gmail.com \\ ${ }^{2}$ West Visayas State University, Iloilo City, Philippines, bobby.gerardo@gmail.com \\ ${ }^{3}$ Technological Institute of the Philippines, Quezon City, Philippines, ruji.medina@tip.edu.ph
}

\begin{abstract}
The demand for food rises proportionally as population grows. To be able to achieve sustainable supply of livestock products, efficient farm management is a necessity. With the advancement in technology it also brought innovations that could be harness in order to achieve better productivity in animal production and agriculture. Precision Livestock Farming (PLF) is a budding concept of making use of smart sensors or available devices to automatically and continuously monitor and manage livestock production. With this concept, this paper introduces a swine management system that integrates image processing technique for weight monitoring. This system captures pig images using camera, evaluate and estimate the weight base on the captured image. It is comprised of several management modules to help swine farm administrators better understand the performance and situation of the swine farm. This paper aims to improve the management in both small and big livestock raisers. Based on the experiment comparing the actual weight and the computed image weight using pixel, there is an average margin of error of $\pm 0.041 \%$ and it clearly shows that it is a good alternative to use the system to avoid the stress of weighing the livestock.
\end{abstract}

Key words : image processing, precision livestock farming, swine management, swine systems, thresholding, weight monitoring

\section{INTRODUCTION}

The pig farmers of today are well-informed than those of the pig farmers of the previous times. In the Philippines, pigs are one of the major livestock entities produced which are then followed by chickens and cattle. According to the [1] , the total hog production in 2012 is more or less be of 1,973.62 thousand metric tons live weight pig. Pigs are just as similar to rice and chicken, which is a staple food used in the Philippines.
Several researches estimate that there will 9 billion people to [2]. For the next 40 years the demand for livestock products will continue to grow [3] and in order to meet this demand, there is a need of more livestock production through expansion of farms.

As demand of livestock such as pig and the number of production increases this makes heavier demands on farmers management skill in order to maintain profits and guarantee the continuity of farms[4]. Thus, this poses challenges in effective swine monitoring and management.

Precision livestock farming (PLF) is an infant technology [5] that combines automatic monitoring and data analysis of animals variables that help increase farmers productivity. Berckman [6] states that the main intention of PLF research and development efforts is to introduce process control principles in agriculture/animal production and management. It is usually a set of interlinked processes that acts together and utilizes measurement, prediction and data analysis of animal variables (e.g. weight, activity, behavior, drinking and feeding behavior, feed intake, sound production and physiological variables) in a continuous and fully automatic way[6].

One of the areas of PLF that needs improvement is monitoring the weight of pigs where traditional backyards farmers either use weighing scales or length or girth measurement to estimate. These method usually require human intervention which result to waste of time, increase in labor and stress to animals and people involved [7]. Other study [8] proposed a method on estimation of live weight of broiler using digital image analysis. Several researches had also utilized image processing in improving management of swine farms [9] [10].

Thresholding is an image processing method used to convert a grey scale image, the value of pixels ranging from $0-255$ into binary image and the value of pixels can have only 2 values 0 or 1 . Thresholding techniques are mainly used in segmentation. The simplest thresholding methods replace 
each pixel in an image with a black pixel if the pixel intensity is less than some fixed constant $\mathrm{T}$, else it is replaced with a white pixel[11].

Image thresholding is a very important problem in automated image analysis. Many image processing and computer vision applications usually require binary images (i.e. black and white) as an introductory step in order to do further processing. By choosing a particular intensity value as "threshold", images can be segmented by setting those pixels whose original intensity is above the threshold as "white pixels", and setting the other pixels as "black pixels." Thresholding is one of the easiest methods to automatically segment an image using a computer[12].

This idea leads the researcher to propose a swine management system using precision livestock farming with image processing that comprise of several modules that ensures effective monitoring and management of swine in the farm. It incorporate image processing as method of weight monitoring to effectively monitor and analyze pig data. The researcher discussed the background and related works in section II and present the overview and components of the system in section III. Section IV shows the implementation and section $\mathrm{V}$ concludes the paper.

\section{BACKGROUND AND RELATED WORKS}

In this section the researcher review several available swine management system that are used by large swine farms. Table 1 shows the capabilities of the software's being available in

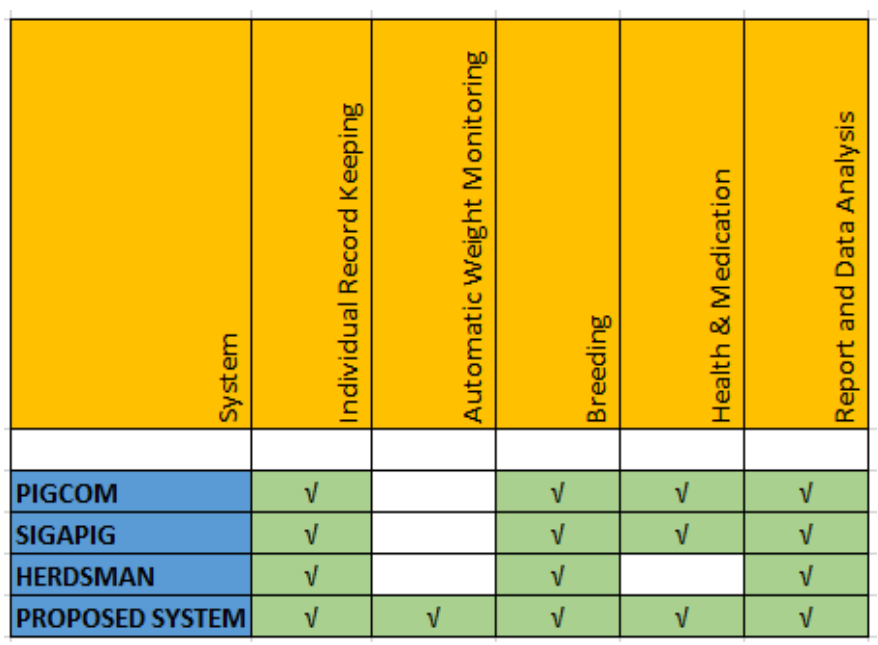

the market which primary task is to record and monitor the weight of the pig. On the other hand, the PIGCOM, SIGAPIG and HERDSMAN have record keeping capabilities. However, there is no automatic weight monitoring using image processing technique.

Table 1: Comparison of Swine Management based on Function

\subsection{PIGCOM}

PigCom is an advanced database management system that allows extensive, detailed recording of every aspect in the life of every sow, boar and feeder in-herd. PigCom allows the user to have an accurate overview of the herd at any time. The performance of any animal can be instantly accessed by its ID. There are weekly reminders \& checklists to take on farm. There are extensive filtering and sorting of information to tailor reports and highlight relevant issues. There is comprehensive selection of weekly, monthly and analytical reports. Farm data can be consolidated to give an overview for larger producers. Graphs and charts bring data to life, showing trends over time and forecasting for the future [13].

\subsection{SIGAPIG}

SigaPig is a software tool for complete swine herd management. It includes easy management of all breeding events in one screen, customizable automated breeding calendar, complete management of treatment and interventions, management of animals by location, genealogy and progeny genetic modules available for purebred facilities. It also manages finishing, feed formulation, genetics and it can generate reports [14].

\subsection{HERDSMAN}

Herdsman is a software program that, in its simplest form, collects data from swine farms and summarizes that data on-farm, into standard reports and action lists to better manage the farm. It has the capacity to create individual animals at farrowing and link them to their parents. You can therefore track multigenerational lineage of inbreeding. In this pedigree analysis you can track 4 reproductive traits and 3 performance traits on each animals and its relationship to the mean of its contemporaries [15].

\section{SYSTEM OVERVIEW AND COMPONENTS}

The System is comprised of different modules namely, Pig, Breeding, Health and Medication, Weight and Data Analysis. It determines the weight of the pigs using camera and reports can be generated and analyzed for decision making. Figure 1 shows the system architecture of the swine management system.

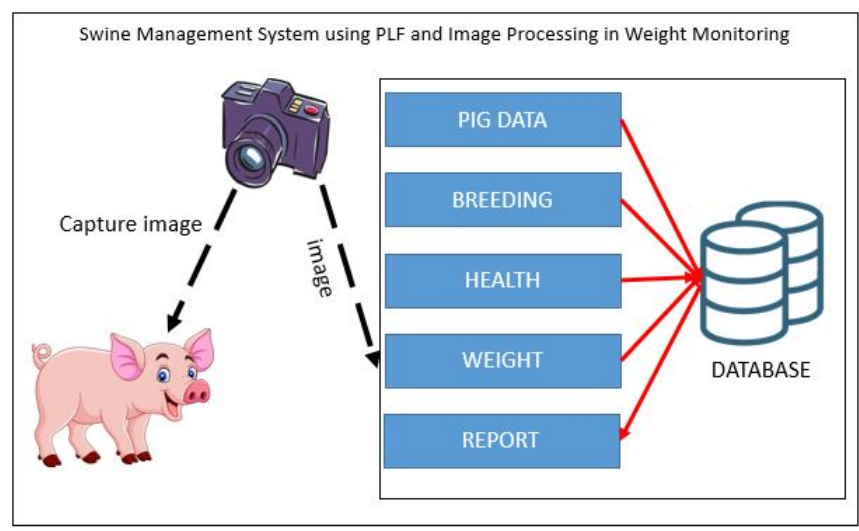

Figure 1: System Architecture 
Anrem J. Balontong et al., International Journal of Advanced Trends in Computer Science and Engineering, 9(1.1), 2020,6 -11

\section{A. Pig Module}

This module includes functionalities like adding, editing, deleting and searching of pig records.

\section{B. Breeding Module}

This module records the breeding data of sows and boars used. Just like the pig module it has the capabilities to insert, edit, delete and search pig records.

\section{Health Medication Module}

This module records the medication and supplements administered to the pigs.

\section{Weight Module}

This module monitors the weight of the pigs using camera. The researcher implement parallel processing using time interval of two times for weight calculation of pigs and the steps for the process are as follows:

a) When the pig goes to drink the camera will capture an image. The captured image will be processed in the first timer by extracting and cropping the big object and store it in the database. Detected small objects (e.g. food, molds, stones, etc.) on the other hand are discarded.

b) Images are then processed by a second timer in a first in first out (FIFO) queue. The images are converted into grayscale followed by application of certain threshold to make it black and white.

c) The system will then check the pig id of the animal using object character recognition (OCR). OCR is a method of converting text image into characters. The pig id are sprayed in the pigs body using a specific swine farm numbers. Authors in [16] also used this method in swine identification. This spray does not cause harm to humans and animals and is a cheap method. The sprayed id number is not permanent but will last for 200 days which would be enough since pigs are mostly sold after 150 days at maximum.

d) Pig ids that were not identified are discarded and those that are identified will be verified in the database if it has already been monitored within an hour. If not, the image will be processed for weight monitoring. Since threshold was applied (in step b) only the black and white pixels will remain in the captured image. The white pixel between the heap and the shoulder of the pig will be counted and converted to centimeter in order to determine the surface area and as basis of the weight of the pig. Then using this information the estimation of weights pig will be handled by the system.

e) After the pig's weight is determined it will be stored in the database along with the corresponding pig id.

As illustrated in figure 2 the image processing will focus on the implementation of dilation and erosion process, during the erosion, the structuring element will scan through the input image without overpass the input image. When all the locations of a pixel value of one on the structuring element overlap with the pixel value of one on particular areas of the input image, the erosion will erode that area and a value of one is set at the origin. The structuring element will scan from left to right, starting at the top left and ending at the bottom right of the input image. During the dilation, the structuring element will scan through the input image, where, at some point, the structuring element will overpass the input image. When any pixel with a value of one on the structuring element overlaps with a pixel value of one on the input image, that particular area of the image will be dilated and a value of one is set at the origin. The structuring element will scan from left to right, starting at the top left and end at the bottom right of the input image.

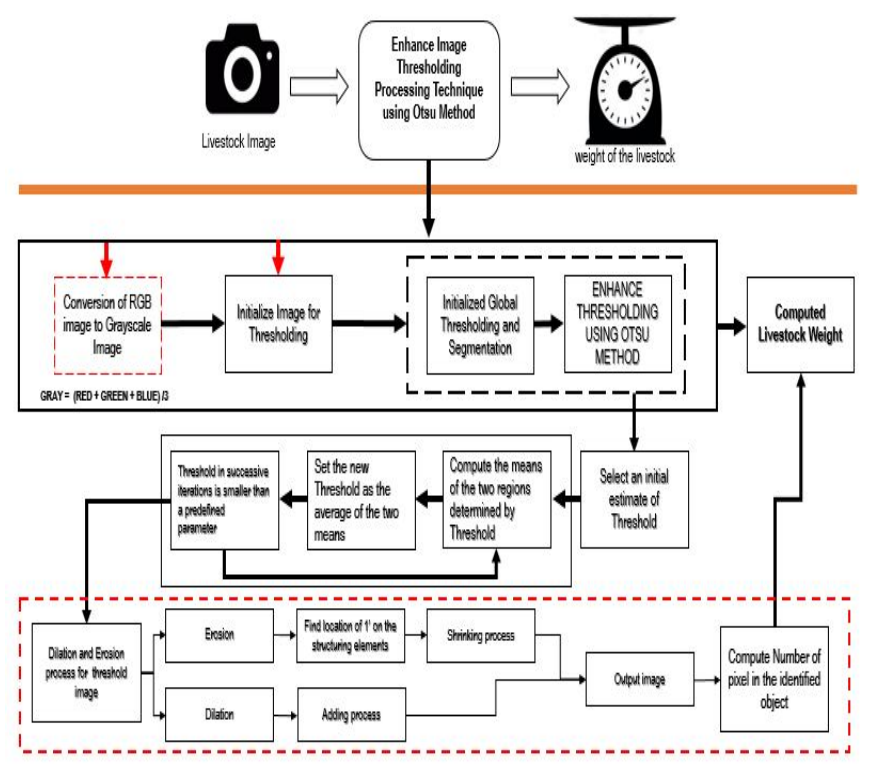

Figure 2: Image Processing Technique

The enhanced threshold method is illustrated in the following steps:

\section{Input: GREY SCALE IMAGE / RGB IMAGE Output: BINARY IMAGE with Computed Weight}

Step 1: Compute histogram and probabilities of each intensity level. 
Anrem J. Balontong et al., International Journal of Advanced Trends in Computer Science and Engineering, 9(1.1), 2020,6 -11

Step 2: Initialized Global Thresholding and Initial Threshold Image

Step 3: compute the 1 class means.

Step 4: Step through all possible thresholds maximum intensity.

Step 5: Update

Step 6: Dilation and Erosion process

Step 7: Compute between class variance

Step 8. Desired threshold corresponds to the maximum value of between class variance

Step 9: Computed Number of Pixel

Step 10: Get the Estimated Weight of the Livestock

\section{E. Data Analysis Module}

This Module analyzes pig data using decision support rules and provide reports by consolidating several pig data like total number of sow, total number of piglets produce, mortality rates, gender and etc.

\section{SYSTEM IMPLEMENTATION}

The System was develop using Microsoft Visual C\# for frontend and MySQL database for the backend. The system is composed of different modules that works together to achieve the systems objective. These modules include the pig, breeding, health and medication, weight, pedigree and data analysis.

Figure 3 shows the Pig Module Screen where users can add, edit, and delete a record of a pig. Data that can be added includes PigID, Name, Breed, Boar, Sow, Birth Date, Gender, Location and weight. A search capability is added to the module for easy access of pig record stored in the system.

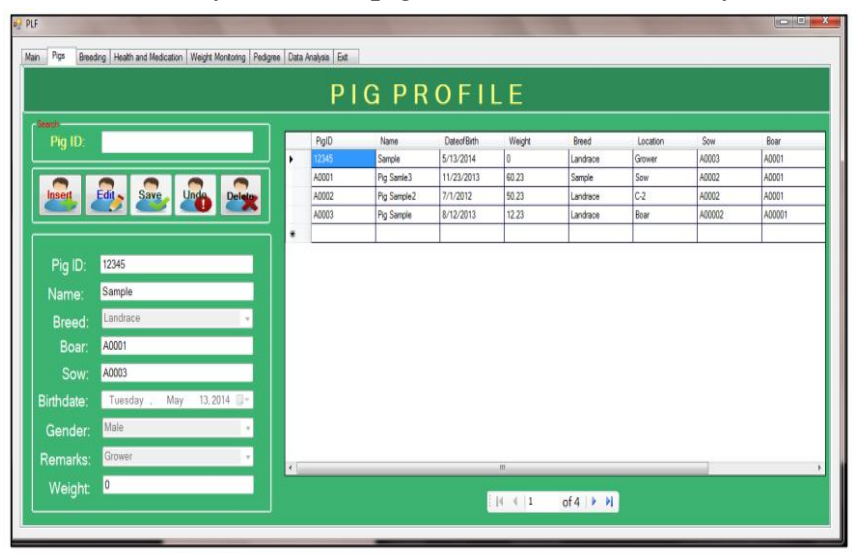

Figure 3: Pig Module

The breeding data of the sow and boar (divided by tabs) can be seen on the Breeding Module Tab shown in Figure 4. Just like the Pig Module Screen, this component lets you also add, edit, delete and search pig records based on breeding parameters such as breeding number, mortality, number of piglets. The breeding data will provide insights about the success of breeding program.

Figure 4: Breeding Module

Figure 5: Health and Medication
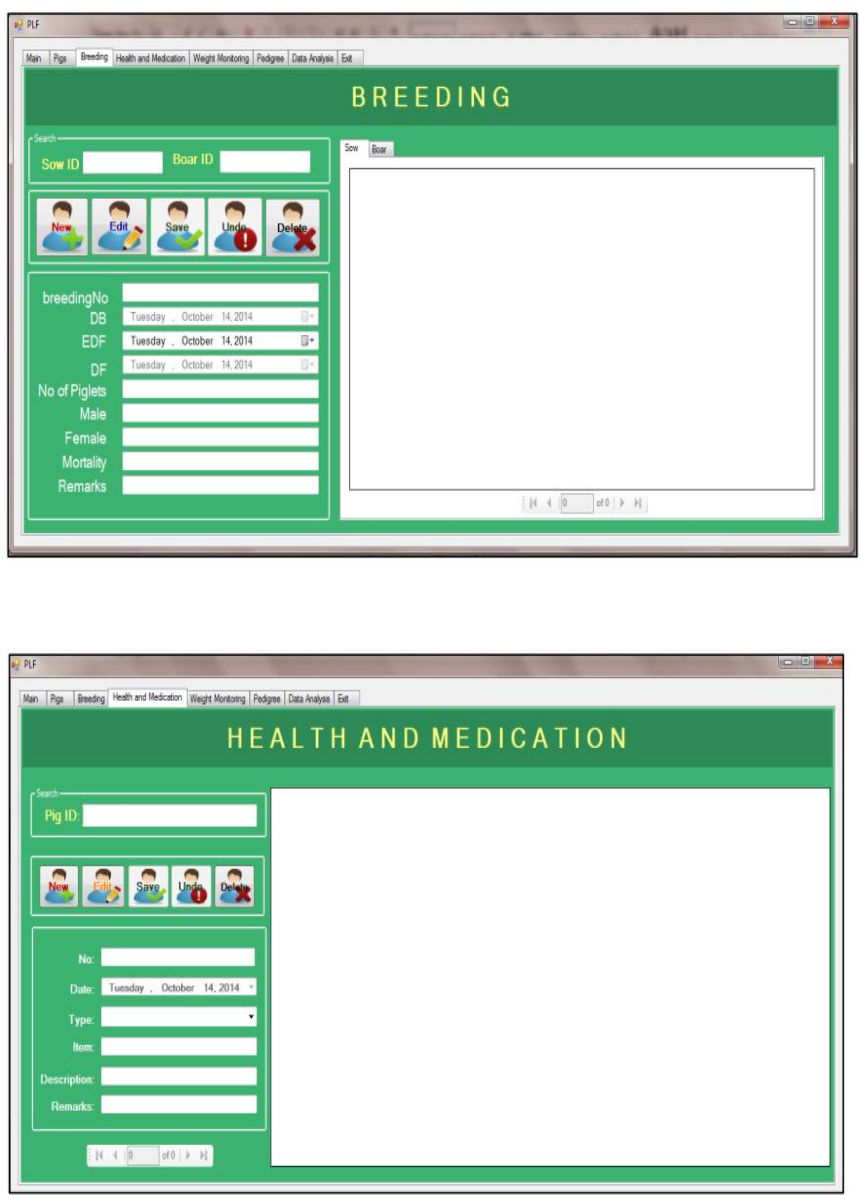

The health and medication module shown in Figure 5 is used to record the medication and supplements administered to the pigs. Figure 6 depicts the weight monitoring screen where user does not need to input anything since weight identification is automatically done by the system using image processing technique as describe in the weight module under section III.

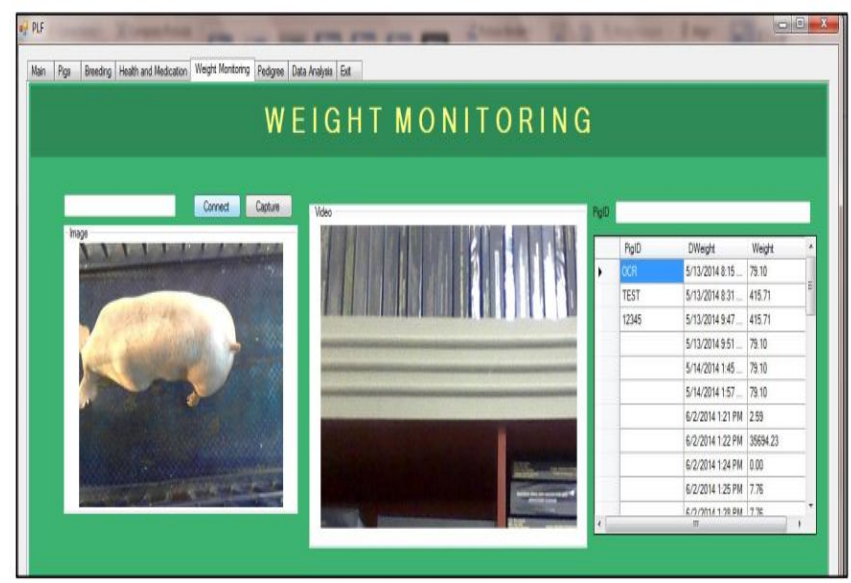

Figure 6: Weight Monitoring 
Figure 7: Report Module

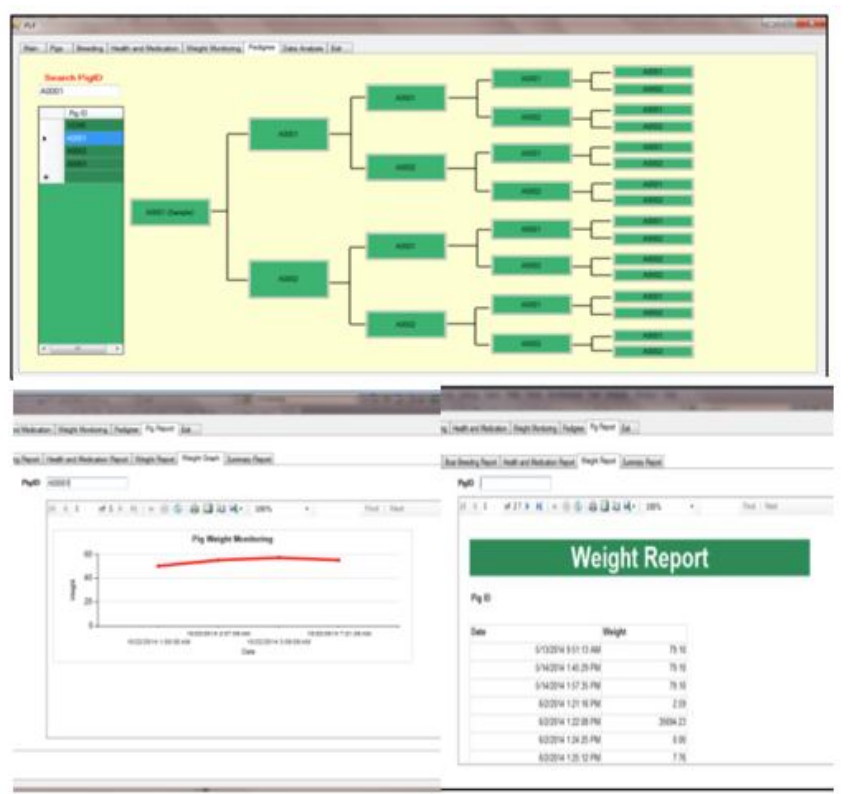

Several reports are generated by the system to help aid administrators in decision making these reports include pedigree analysis, pig report, pedigree analysis, and weight trends.

\section{DESIGN AND IMPLEMENTATION ISSUES}

Several issues had been encountered during the development of the system. The first issue was how to identify the pigs based on their location. Several methods had been used. One of them was the use of RFID. The use of RFID is very expensive. It cannot also give the precise location of the pig. The alternative was the use of OCR or object character recognition. Using this method pigs can be easily identified no matter where they move. Another problem that was encountered was to capture the body of the pigs perfectly. Pigs move their body very often and the only time that you can capture their image is when they go for a drink or eat their feeds. So the perfect area for monitoring the pig is at the drinking area or the feeding area.

\section{RESULT AND DISCUSSION}

The pigs were weighed using digital weighing scale and at the same time the images were captured using camera at a distance of $6 \mathrm{ft}$. and 6 inches. The images were numbered according to the weight of the pigs and store in the system. First filtering was applied to get the noise from each image. The noises are errors in image acquisition. Grey-scaling was then applied to convert a continuous-tone image to an image that can be manipulated by a computer.
The image was compared to a pig template using template matching. The matched image template was then cropped and converted into black and white. The white pixels are then counted in the black and white image [17]. The number of white pixels was used to get the weight of the pig using the surface law[7] $\quad(\mathrm{S}=\alpha \mathrm{W} 2 / 3$; where $\mathrm{S}=$ surface area, $\alpha=$ constant, $\mathrm{W}=$ weight) . The Formula to get the weight of the pig is $\mathrm{W}=\mathrm{S} / \alpha 2 / 3(\mathrm{~S}=$ no of white pixel $\alpha=$ constant and $\mathrm{W}$ = weight).

As Illustrated in the table 2 in this test, there is an average margin of error of $\pm 0.041 \%$ on using the number of pixels to compute the weight of the livestock. As a summary it clearly shows that the use of camera in estimating the weight of the pig can be a good alternative to avoid the stress of weighing.

Table 2: Result of the actual weight and capture image weight

\begin{tabular}{|c|c|c|c|}
\hline Actual Weight & Camera Weight & Difference & Percentage \\
\hline 79 & 79.02305358 & -0.023054 & -0.0291817 \\
\hline 77 & 76.99894946 & 0.0010505 & 0.0013643 \\
\hline 81 & 81.00105055 & -0.001051 & -0.001297 \\
\hline 85 & 84.99854092 & 0.0014591 & 0.0017166 \\
\hline 80 & 80.00052528 & -0.000525 & -0.0006566 \\
\hline 78 & 77.99947473 & 0.0005253 & 0.0006734 \\
\hline 85 & 84.99854092 & 0.0014591 & 0.0017166 \\
\hline 85 & 85.25213027 & -0.25213 & -0.2966238 \\
\hline 77 & 77.06811019 & -0.06811 & -0.0884548 \\
\hline 82 & 82.00157582 & -0.001576 & -0.0019217 \\
\hline Average & & -0.0412665 \\
\hline
\end{tabular}

\section{CONCLUSION}

The demand for food supply increase rapidly as population grows. To be able to cope with demands, expansion of livestock farm are necessary. As the PLF continue to mature, more farmers will benefit from this technology. In this research the automatic weight, monitoring was integrated in the pig farm management to form a PLF. Through the use of PLF it would be easy for the farmers to monitor and manage their farm. The automatic weight monitoring will reduce the burden of farmers in monitoring the growth of the pigs. They can also efficiently access valuable and accurate information anytime they want. This valuable information can help them in their decision-making and will help in increasing livestock production. 


\section{ACKNOWLEDGEMENT}

The authors would like to acknowledge Western Institute of Technology for the Support. Technological Institute of the Philippines Graduate School Dean and to my Adviser Dr. Bobby Gerardo for the guidance and knowledge imparted. To God, my family and friends.

\section{REFERENCES}

1. Bureau of agriculture statistics, "Statistics on Agriculture," Philippines, no. 742, 2013.

2. M. C. T. Scholten, I. J. M. De Boer, B. Gremmen, and C. Lokhorst, "Livestock Farming with Care: Towards sustainable production of animal-source food," NJAS Wageningen J. Life Sci., vol. 66, pp. 3-5, 2013. https://doi.org/10.1016/j.njas.2013.05.009

3. a McLeod and N. F. and A. O. of the United, World Livestock 2011 Livestock in food security World. 2011.

4. J. POMAR and C. POMAR, "A knowledge-based decision support system to improve sow farm productivity," Expert Syst. Appl., vol. 29, no. 1, pp. 33-40, Jul. 2005.

5. "The Royal Veterinary College, University of London, Hatfield, Hertfordshire, AL9 7TA, United Kingdom," Production, pp. 397-404, 2007.

6. D. Berckmans, "Automatic on-line monitoring of animals by precision livestock farming," Int. Soc. Anim. Hyg., no. April, pp. 1-307, 2006.

7. L. E. O. Zaragoza, "Evaluation of the accuracy of simple body measurements for live weight prediction in growing-finishing pigs," 2009.

8. M. B. R. Mollah, M. A. Hasan, M. A. Salam, and M. A. Ali, "Digital image analysis to estimate the live weight of broiler," Comput. Electron. Agric., vol. 72, no. 1, pp. 48-52, Jun. 2010. https://doi.org/10.1016/j.compag.2010.02.002

9. M. Kashiha et al., "Automatic weight estimation of individual pigs using image analysis," Comput. Electron. Agric., vol. 107, pp. 38-44, 2014.

10. M. Kashiha et al., "The automatic monitoring of pigs water use by cameras," Comput. Electron. Agric., vol. 90, pp. 164-169, Jan. 2013.

https://doi.org/10.1016/j.compag.2012.09.015

11. "Computer Vision Talks: OTSU thresholding." [Online]. Available:

http://computervisionwithvaibhav.blogspot.com/2015/1

0/otsu-thresholding.html. [Accessed: 24-Oct-2018].

12. Process Dynamic Solutions, "Chapter 3," Pulse, vol. 1, pp. 1-26, 2004.

13. "D2D PigCom Software." [Online]. Available: http://www.pigcom.co.uk/. [Accessed: 14-Dec-2019].

14. "A range of farm software for swine herd management SIGA." [Online]. Available: https://www.siga.net/en-ca/swine-herd-operations\#_Fac ets@_IdentifierGuid=395cdcbb-fa25-6d7c-a102-8619fd
ff80c7\&PropertyName:0\&ValueType=Value\&Values@ =4f9f6a1c-4779-0476-dc98-bbc0b884ab10;\&IdentifierG uidType $=3 ; ; \&$ Search $=\&$ OrderBy:null\&IsDescOrder:fals e\&Index: [Accessed: 15-Dec-2019].

15. "Herdsman | Home." [Online]. Available: http://www.herdsman.com/. [Accessed: 15-Dec-2019].

16. M. Kashiha et al., "Automatic identification of marked pigs in a pen using image pattern recognition," Comput. Electron. Agric., vol. 93, pp. 111-120, Apr. 2013. https://doi.org/10.1016/j.compag.2013.01.013

17. A. Martin and S. Tosunoglu, "Image Processing Techniques For Machine Vision," Miami, Florida, pp. $1-9,2000$. 